TIT/HEP-520

hep-th/0405161

May, 2004

\title{
Effective Theory on Non-Abelian Vortices in Six Dimensions
}

\author{
Minoru Eto *, Muneto Nitta ${ }^{\dagger}$ and Norisuke Sakai ${ }^{\ddagger}$ \\ Department of Physics, Tokyo Institute of Technology \\ Tokyo 152-8551, JAPAN
}

\begin{abstract}
Non-Abelian vortices in six spacetime dimensions are obtained for a supersymmetric $U(N)$ gauge theory with $N$ hypermultiplets in the fundamental representation. Massless (moduli) fields are identified and classified into Nambu-Goldstone and quasi-Nambu-Goldstone fields. Effective gauge theories for the moduli fields are constructed on the fourdimensional world volume of vortices. A systematic method to obtain the most general form of the effective Lagrangian consistent with symmetry is proposed. The moduli space for the multi-vortices is found to be a vector bundle over the complex Grassmann manifold.
\end{abstract}

\footnotetext{
*e-mail address: meto@th.phys.titech.ac.jp

$\dagger$ †-mail address: nitta@th.phys.titech.ac.jp

‡e-mail address: nsakai@th.phys.titech.ac.jp
} 


\section{Introduction}

The brane-world scenario [1, 2, 3] has attracted much attention in recent years. These models with extra dimensions require some topological defects in higher dimensional spacetime. It is often advantageous to consider supersymmetric (SUSY) models in order to construct such topological defects. The topological defects typically break part of the original SUSY [4] leaving the $\mathcal{N}=1$ SUSY in four-dimensional world volume of the topological defect. In this way we may be able to obtain realistic unified models of the type of the minimal SUSY standard model [5].

Since standard model particles should be realized as low-energy fluctuations localized on topological defects such as walls and vortices, it is important to study modes which are massless or nearly massless compared to the mass scale of the topological defects. When we consider a solution of the equations of motion as a possible background corresponding to the topological defect, it can often contain parameters. These parameters are called moduli and represent possible deformations of the background solution without costing energy. Therefore one can promote the parameters into fields on the world volume of the topological defect [6]. These fields are massless and are called moduli fields. The moduli fields describe the low-energy dynamics of the topological defect. Some of the massless fields may originate from spontaneously broken global symmetries and are called NambuGoldstone particles. Number of the Nambu-Goldstone particles are given by the number of broken symmetry generators including the spacetime symmetry generators.

Low-energy interactions of the Nambu-Goldstone particles are severely constrained by the low-energy theorems which may be obtained by the method of nonlinear realization [7]. On the other hand, there may be massless particles which do not correspond to the spontaneously broken generators. In the case of SUSY theories where $\mathcal{N}=1$ SUSY is maintained in four-dimensions, scalar particles have to form complex fields in order to form a chiral scalar field. If a Nambu-Goldstone particle does not form a complex scalar with another Nambu-Goldstone scalar, it still has to accompany a massless scalar to form a chiral scalar field. Such massless scalars do not correspond to broken symmetry generators and are required to exist only because of SUSY. These massless scalars are called quasiNambu-Goldstone bosons [8]-[14], and will acquire masses once SUSY is broken. For a model building, it is extremely useful to obtain Nambu-Goldstone particles as well as quasi-Nambu-Goldstone particles to obtain particles in the low-energy effective theories.

Walls are the simplest topological defects with single extra dimension. Walls with eight SUSY, whose world-volume theories preserve four SUSY, were discussed in [15]. 
If we have two extra dimensions, we need to have topological defects such as vortices. Vortex is a typical topological defect with codimension two. In the pioneering work of Abrikosov [16], and Nielsen and Olsen [17] they have worked out a vortex in the $U(1)$ gauge theory, which is called the ANO vortex. Vortices with higher vorticities [18] and vortices in higher dimensions [19] have also been constructed. In the brane-world scenario, vortices are especially useful to localize gauge fields using the warped compactifications [20]. More recently, monopoles in the Higgs phase have been found [21]-27] in the three- or fourdimensional SUSY gauge theories with eight SUSY. They can be static solutions when they are attached to non-Abelian vortices at both sides [23. These non-Abelian vortex solutions are obtained by deforming the $U(1)$ ANO vortex embedded into the SUSY non-Abelian gauge theories. It has been observed that the non-Abelian vortices have interesting characteristics compared to Abelian vortices. Non-Abelian monopoles in the Higgs phase are also discussed in the context of monopole confinement by the $Z_{k}$ vortex in the deformed $\mathcal{N}=2$ and $\mathcal{N}=4$ SUSY Yang-Mills theories 28].

The number of moduli fields can be counted by means of index theorems for many topological defects, such as vortices [29]. More recently, new methods have been proposed to construct effective theories of moduli fields on the world volume of the topological defect, such as vortices or instantons [21, 27]. One of the methods uses a field theoretical Ansatz 22], which becomes simple for a particular case where the $U(1)$ gauge coupling $e$ is related to the $S U(N)$ gauge coupling $g$ by $g^{2}=N e^{2}$ [21]. The other method is inspired by brane constructions and gives a gauge theory on the world volume. Both of them have been applied to the three-dimensional $U(N)$ gauge theories with eight SUSY, leading to gauge theories with four SUSY in the two-dimensional world volume of the vortices [21]. Although they have considered a particular case between coupling constants, the results are expected to apply to other cases (with some deformations of moduli metric and so on). Since there are ambiguities to define the moduli fields, one can have different metric of moduli fields in various approaches for low-energy dynamics of topological defects such as vortices. In particular it has been noted that the moduli metric obtained by using the method of Manton [6] is different from that deduced from the world-volume gauge theory inspired by brane constructions [21].

The purpose of this paper is to discuss non-Abelian BPS vortex solutions in a sixdimensional $U(N)$ SUSY gauge theory with $N$ hypermultiplets in the fundamental representation and to work out the effective field theories for their moduli fields on the four-dimensional world volume, so that it may be useful for a model building in the brane-world scenario. We work out BPS equations for multi-vortex solutions and con- 
struct their moduli space. We succeed to distinguish the Nambu-Goldstone fields and quasi-Nambu-Goldstone fields. We also obtain general form of the effective field theories for the moduli fields in the spirit of the world-volume gauge theories inspired by brane constructions [21]. Since the effective theories on the BPS vortices have four SUSY, we obtain Kähler quotient construction for the Grassmann manifold as the moduli space. We also work out possible forms of the metric consistent with the symmetry requirement of the low-energy effective theory. This will hopefully explain the discrepancies [21, 6] noted previously between two different methods to derive the low-energy effective theory. Similarly the deformations of the moduli metric in the case of $g^{2} \neq N e^{2}$ may be also incorporated by this method.

In sect 2, the six-dimensional Lagrangian with eight SUSY is constructed using the superfield representing four SUSY, at the cost of sacrificing the manifest Lorentz invariance in six dimensions. In sect 3, non-Abelian vortices are constructed in six dimensions. In sect 4. effective theory on the world volume of vortices is constructed. In sect 5, general form of deformations of vortex moduli space is studied. Sect 6 is devoted to a discussion of possible uses of our results in the brane-world context.

\section{$2 D=6$ Lagrangian in 4 SUSY Superspace}

We start with six-dimensional $\mathcal{N}=1$ (i.e. eight supercharges) supersymmetric (SUSY) gauge theory with the $U(1) \times S U\left(N_{\mathrm{C}}\right)$ gauge group and $N_{\mathrm{F}}$ hypermultiplets with the same (unit) $U(1)$ charges. We are especially interested in the case of $N_{\mathrm{C}}=N_{\mathrm{F}} \equiv N$. We will study non-Abelian vortices in six-dimensions, which lie in the $x^{5}-x^{6}$ plane and have four-dimensional world-volume extending from $x^{0}$ to $x^{3}$ applicable to the brane-world scenario. For this purpose, it is useful to express the $D=6$ Lagrangian in terms of 4 SUSY superfield formalism discussed in [30]. At the cost of sacrificing the manifest $D=6$ Lorentz invariance, we can use this superfield formalism, maintaining the $D=4$ Lorentz invariance. We first review their formalism briefly in this section.

Each $D=6$ supermultiplet is decomposed into a set of 4 SUSY superfields (with dependence of $x^{5}$ and $x^{6}$ coordinates) as follows. The $U(1)$ vector multiplet is decomposed into $(V, \Phi)$ with $V$ and $\Phi$ vector and chiral superfields, respectively, in 4 SUSY superfield formalism. A set of $N$ by $N$ matrix superfields $(\hat{V}, \hat{\Phi})$ constitutes $D=6 S U(N)$ vector multiplets. Here a hat denotes a matrix in the fundamental representation of $S U(N)$ 
which can be decomposed in terms of basis $T^{A}$

$$
\hat{X}_{b}^{a}=\sum_{A=1}^{N^{2}-1} X^{A}\left(T^{A}\right)^{a}{ }_{b}
$$

where $A$ is the adjoint index running $1, \cdots, N^{2}-1$, and $a, b$ are indices of the fundamental representation running $1, \cdots, N_{\mathrm{C}}=N$. The $N_{\mathrm{F}}$ hypermultiplets in the fundamental representation are decomposed into $\left(Q_{i}, \tilde{Q}^{i}\right)\left(i=1, \cdots, N_{\mathrm{F}}=N\right)$ with $Q_{i}, \tilde{Q}^{i}$ chiral superfields belonging to the fundamental and the anti-fundamental representations of $S U(N)$, respectively, with the opposite $U(1)$ charges. Here we have denoted $Q_{i}$ and $\tilde{Q}^{i}$ by column and row vectors whose components are denoted by $a$, but sometimes we use matrix notations, $Q^{a}{ }_{i}$ and $\tilde{Q}^{i}{ }_{a}$.

Let us denote the six-dimensional indices by capital letters $M=0,1,2,3,5,6$, and four-dimensional indices by small letters $m=0,1,2,3$. We define the covariant derivative

$$
\mathcal{D}_{M} \equiv \partial_{M}-\frac{i}{2} A_{M}-\frac{i}{2} \hat{A}_{M}
$$

We can decompose vector fields $A_{M}, \hat{A}_{M}$ in six dimensions to vector fields $A_{m}, \hat{A}_{m}$ and complex scalar fields $\phi, \hat{\phi}$

$$
\phi=\frac{A_{6}+i A_{5}}{\sqrt{2}}, \quad \hat{\phi}=\frac{\hat{A}_{6}+i \hat{A}_{5}}{\sqrt{2}} .
$$

The field strengths and covariant derivatives in extra dimensions can then be rewritten ${ }^{1}$

$$
\begin{aligned}
F_{56} & =\frac{1}{\sqrt{2}}(\partial \bar{\phi}+\bar{\partial} \phi), \\
\hat{F}_{56} & =\frac{1}{\sqrt{2}}\left(\partial \hat{\phi}^{\dagger}+\bar{\partial} \hat{\phi}+\frac{1}{\sqrt{2}}\left[\hat{\phi}^{\dagger}, \hat{\phi}\right]\right), \\
\mathcal{D} q_{i}^{a} & =\partial q_{i}^{a}-\frac{1}{\sqrt{2}} \phi q^{a}{ }_{i}-\frac{1}{\sqrt{2}} \hat{\phi}^{a}{ }_{b} q^{b}{ }_{i}, \\
\overline{\mathcal{D}} \tilde{q}_{i}^{\dagger a} & =\bar{\partial} \tilde{q}^{\dagger a}{ }_{i}+\frac{1}{\sqrt{2}} \bar{\phi} \tilde{q}^{\dagger a}{ }_{i}+\frac{1}{\sqrt{2}} \hat{\phi}^{\dagger a}{ }_{b} \tilde{q}^{\dagger b}{ }_{i} .
\end{aligned}
$$

Invariance under gauge transformations requires a Wess-Zumino-Witten term in general gauges [31, 30]. In the Wess-Zumino gauge, superfields can be expanded in terms of

\footnotetext{
${ }^{1}$ We denote complex conjugate by bar and hermitian conjugate for matrix by ${ }^{\dagger}$.
} 
component fields as

$$
\begin{aligned}
V\left(x^{m}, \theta, \bar{\theta} ; x^{5}, x^{6}\right) & =-\theta \sigma^{m} \bar{\theta} A_{m}+i \bar{\theta} \theta^{2} \lambda_{1}-i \theta^{2} \bar{\theta} \bar{\lambda}_{1}+\frac{1}{2} \theta^{2} \bar{\theta}^{2}\left(D-F_{56}\right), \\
\Phi\left(y^{m}, \theta ; x^{5}, x^{6}\right) & =\phi+\sqrt{2} \theta \lambda_{2}+\theta^{2} F_{\Phi}, \\
\hat{V}\left(x^{m}, \theta, \bar{\theta} ; x^{5}, x^{6}\right) & =-\theta \sigma^{m} \bar{\theta} \hat{A}_{m}+i \bar{\theta} \theta^{2} \hat{\lambda}_{1}-i \theta^{2} \bar{\theta} \bar{\lambda}_{1}+\frac{1}{2} \theta^{2} \bar{\theta}^{2}\left(\hat{D}-\hat{F}_{56}\right), \\
\hat{\Phi}\left(y^{m}, \theta ; x^{5}, x^{6}\right) & =\hat{\phi}+\sqrt{2} \theta \hat{\lambda}_{2}+\theta^{2} \hat{F}_{\Phi}, \\
Q_{i}\left(y^{m}, \theta ; x^{5}, x^{6}\right) & =q_{i}+\sqrt{2} \theta \psi_{i}+\theta^{2}\left(F_{i}+\overline{\mathcal{D}} \tilde{q}_{i}^{\dagger}\right), \\
\tilde{Q}^{i}\left(y^{m}, \theta ; x^{5}, x^{6}\right) & =\tilde{q}^{i}+\sqrt{2} \theta \tilde{\psi}^{i}+\theta^{2}\left(\tilde{F}^{i}-\overline{\mathcal{D}} q^{\dagger i}\right) .
\end{aligned}
$$

Here $x^{m}(m=0,1,2,3)$ are the $D=4$ spacetime coordinates and $y^{m}$ are the chiral coordinates defined by $y^{m} \equiv x^{m}+i \theta \sigma^{m} \bar{\theta}$ with $\theta(\bar{\theta}) D=4$ two-component Weyl (antiWeyl) spinor coordinates in the 4 SUSY superspace. All component fields in the right hand sides depend on $x^{m}, x^{5}$ and $x^{6}$. Fermions $\lambda_{1}, \lambda_{2}, \hat{\lambda}_{1}, \hat{\lambda}_{2}, \psi_{i}$ and $\tilde{\psi}^{i}$ transform as $D=4$ two-component Weyl spinors but depend on extra coordinates also. The gauginos $\lambda_{1}, \lambda_{2}, \hat{\lambda}_{1}$, and $\hat{\lambda}_{2}$, together form a symplectic Majorana spinor in 6 dimensions, whereas $\psi_{i}$ and $\tilde{\psi}^{i}$ together transform as a $D=4$ Dirac spinor. Note that the last terms in Eqs.(2.7), (2.9), (2.11), and (2.12) are $D$-term and $F$-term components in $V$ and $Q$, but are not $D=6$ Lorentz scalars themselves. Only after subtracting $F_{56}, \overline{\mathcal{D}} \tilde{q}^{\dagger} i$, or $\overline{\mathcal{D}} q^{\dagger i}$, the auxiliary fields $D, \hat{D}, F_{i}, \tilde{F}^{i}$ become genuine $D=6$ Lorentz scalars. In the last two equations we used the holomorphic covariant derivative with respect to extra coordinates defined by

$$
\mathcal{D} \equiv \mathcal{D}_{5}-i \mathcal{D}_{6} .
$$

Taking the Wess-Zumino gauge, we obtain the Lagrangian [30]

$$
\begin{aligned}
\mathcal{L}= & \operatorname{Tr}\left[\frac{1}{4 g^{2}}\left(\int d^{2} \theta \hat{W}^{\alpha} \hat{W}_{\alpha}+\int d^{2} \bar{\theta} \hat{W}_{\dot{\alpha}}^{\dagger} \hat{W}^{\dot{\alpha} \dagger}\right)\right. \\
& \left.\quad+\frac{1}{g^{2}} \int d^{2} \theta d^{2} \bar{\theta}\left\{\left(\hat{\Phi}^{\dagger}+\sqrt{2} \bar{\partial}\right) \mathrm{e}^{-\hat{V}}(\hat{\Phi}-\sqrt{2} \partial) \mathrm{e}^{\hat{V}}+\bar{\partial} \mathrm{e}^{-\hat{V}} \partial \mathrm{e}^{\hat{V}}\right\}\right] \\
+ & \frac{1}{4 e^{2}}\left(\int d^{2} \theta W^{\alpha} W_{\alpha}+\int d^{2} \bar{\theta} \bar{W}_{\dot{\alpha}} \bar{W}^{\dot{\alpha}}\right) \\
+ & \frac{1}{e^{2}} \int d^{2} \theta d^{2} \bar{\theta}\{(\bar{\Phi}-\sqrt{2} \bar{\partial} V)(\Phi-\sqrt{2} \partial V)-\bar{\partial} V \partial V\} \\
+ & \int d^{2} \theta d^{2} \bar{\theta}\left[Q^{i \dagger} \mathrm{e}^{-V-\hat{V}} Q_{i}+\tilde{Q}^{i} \mathrm{e}^{V+\hat{V}} \tilde{Q}_{i}^{\dagger}\right] \\
+ & \int d^{2} \theta\left[\tilde{Q}^{i}\left(\partial-\frac{1}{\sqrt{2}} \Phi-\frac{1}{\sqrt{2}} \hat{\Phi}\right) Q_{i}+\frac{v^{2}}{2 \sqrt{2}} \Phi\right] \\
+ & \int d^{2} \bar{\theta}\left[Q^{i \dagger}\left(-\bar{\partial}-\frac{1}{\sqrt{2}} \bar{\Phi}-\frac{1}{\sqrt{2}} \hat{\Phi}^{\dagger}\right) \tilde{Q}_{i}^{\dagger}+\frac{v^{2}}{2 \sqrt{2}} \bar{\Phi}\right]
\end{aligned}
$$


where contraction of $S U(N)$ flavor index $i$ is implied, and the normalization of the $S U(N)$ generators is taken as $\operatorname{Tr}\left(T^{A} T^{B}\right)=\delta^{A B}$. The 4 SUSY superfield strengths are defined by

$$
W_{\alpha}=-\frac{1}{4} \bar{D} \bar{D} D_{\alpha} V, \quad \hat{W}_{\alpha}=\frac{1}{4} \bar{D} \bar{D} \mathrm{e}^{\hat{V}} D_{\alpha} \mathrm{e}^{-\hat{V}} .
$$

A real constant $v^{2}$ is called the Fayet-Iliopoulos parameter ${ }^{2}$. A holomorphic coordinate $z$ and the derivative $\partial$ with respect to $z$ are used for coordinates in extra dimensions

$$
z=\frac{1}{2}\left(x^{5}+i x^{6}\right), \quad \partial \equiv \partial_{5}-i \partial_{6},
$$

The Lagrangian (2.14) is invariant under the supergauge transformations, given by

$$
\begin{aligned}
& V \rightarrow V+\Lambda+\bar{\Lambda}, \quad \Phi \rightarrow \Phi+\sqrt{2} \partial \Lambda, \\
& \mathrm{e}^{\hat{V}} \rightarrow \mathrm{e}^{\hat{\Lambda}} \mathrm{e}^{\hat{V}} \mathrm{e}^{\hat{\Lambda}^{\dagger}}, \quad \hat{\Phi} \rightarrow \mathrm{e}^{\hat{\Lambda}}(\hat{\Phi}-\sqrt{2} \partial) \mathrm{e}^{-\hat{\Lambda}}, \\
& Q_{i} \rightarrow \mathrm{e}^{\Lambda+\hat{\Lambda}} Q_{i}, \quad \tilde{Q}^{i} \rightarrow \tilde{Q}^{i} \mathrm{e}^{-\Lambda-\hat{\Lambda}},
\end{aligned}
$$

with $\Lambda(\bar{\Lambda})$ and $\hat{\Lambda}=\sum_{A} \Lambda^{A} T^{A}\left(\hat{\Lambda}^{\dagger}=\sum_{A} \bar{\Lambda}^{A} T^{A}\right)$ chiral (anti-chiral) superfields for $U(1)$ and $S U(N)$ gauge transformations, respectively. Note that $\Phi$ and $\hat{\Phi}$ also receive gauge transformations.

In the Lagrangian (2.14) the $D=6$ kinetic terms of the $U(1)$ and $S U(N)$ gauge fields consist of the four-dimensional part and the extra-dimensional part. The first and third lines of Eq.(2.14) give the four-dimensional part in terms of the ordinary 4 SUSY superfield strengths in Eq.(2.15) which contain ordinary gauge field strengths

$$
F_{m n}=\partial_{m} A_{n}-\partial_{n} A_{m}, \quad \hat{F}_{m n}=\partial_{m} \hat{A}_{n}-\partial_{n} \hat{A}_{m}-\frac{i}{2}\left[\hat{A}_{m}, \hat{A}_{n}\right] .
$$

On the other hand, the extra-dimensional part is given by the fourth line for the $U(1)$ part and the second line for the $S U(N)$ part in the Lagrangian (2.14), respectively,

The auxiliary fields can be eliminated by their algebraic equations of motion as

$$
\begin{aligned}
D & =\frac{e^{2}}{2}\left(q^{\dagger i} q_{i}-\tilde{q}^{i} \tilde{q}_{i}^{\dagger}\right), \quad D^{A}=\frac{g^{2}}{2}\left(q^{\dagger i} T^{A} q_{i}-\tilde{q}^{i} T^{A} \tilde{q}_{i}^{\dagger}\right), \\
F_{\Phi} & =\frac{e^{2}}{\sqrt{2}}\left(q^{\dagger i} \tilde{q}_{i}^{\dagger}-\frac{v^{2}}{2}\right), \quad F_{\Phi}^{A}=\frac{g^{2}}{\sqrt{2}} q^{\dagger i} T^{A} \tilde{q}_{i}^{\dagger}, \\
F_{i} & =0, \quad \tilde{F}_{i}^{\dagger}=0 .
\end{aligned}
$$

\footnotetext{
${ }^{2}$ We choose the F-type ("magnetic") Fayet-Iliopoulos parameter by using $S U(2)_{R}$ rotation.
} 
Substituting these back into the component Lagrangian, we obtain the scalar potential

$$
\begin{aligned}
V= & \frac{1}{g^{2}} \sum_{A=1}^{N^{2}-1}\left(\frac{1}{2}\left(D^{A}\right)^{2}+\left|F_{\Phi}^{A}\right|^{2}\right)+\frac{1}{e^{2}}\left(\frac{1}{2} D^{2}+\left|F_{\Phi}\right|^{2}\right)+F^{\dagger i} F_{i}+\tilde{F}^{i} \tilde{F}_{i}^{\dagger} \\
= & g^{2} \sum_{A=1}^{N^{2}-1}\left[\frac{1}{8}\left(q^{\dagger i} T^{A} q_{i}-\tilde{q}^{i} T^{A} \tilde{q}_{i}^{\dagger}\right)^{2}+\frac{1}{2}\left|q^{\dagger i} T^{A} \tilde{q}_{i}^{\dagger}\right|^{2}\right] \\
& +e^{2}\left[\frac{1}{8}\left(q^{\dagger i} q_{i}-\tilde{q}^{i} \tilde{q}_{i}^{\dagger}\right)^{2}+\frac{1}{2}\left|q^{\dagger i} \tilde{q}_{i}^{\dagger}-\frac{v^{2}}{2}\right|^{2}\right]
\end{aligned}
$$

The supersymmetric vacuum is determined through the $D$-term and $F$-term flat conditions:

$$
q_{i}^{a}=\tilde{q}_{i}^{\dagger a}=\frac{v}{\sqrt{2 N}} \delta^{a}{ }_{i} .
$$

These vacuum expectation values (VEVs) break gauge symmetry to a discrete symmetry $\mathbf{Z}_{N}$ where broken $U(1)$ part ensures the topological stability of vortex configuration

$$
\pi_{1}\left(\frac{S U(N) \times U(1)}{\mathbf{Z}_{N}}\right)=\mathbf{Z} \ni k,
$$

with the topological charge $k$. Simultaneously the VEVs break the global symmetry as

$$
U(1)_{\mathrm{G}} \times S U(N)_{\mathrm{G}} \times S U(N)_{\mathrm{F}} \rightarrow S U(N)_{\mathrm{G}+\mathrm{F}}
$$

where $U(1)_{\mathrm{G}} \times S U(N)_{\mathrm{G}}$ is a global transformation in the gauge symmetry. The unbroken group $S U(N)_{\mathrm{G}+\mathrm{F}}$ acts on $q$ as

$$
q^{a}{ }_{i} \rightarrow U^{a}{ }_{b} q_{j}^{b} U^{-1 j}{ }_{i}
$$

with the same group element $U$ in $S U(N)_{\mathrm{G}}$ and $S U(N)_{\mathrm{F}}$, and hence this vacuum is called the color-flavor locking vacuum.

\section{Non-Abelian Vortices in Six Dimensions}

In this section we construct the vortex configuration which depends on $x^{5}, x^{6}$ only, and review solutions for non-Abelian vortices obtained in [21] and [22] in three and four dimensions, respectively. We will add some details of comparison between solutions for different values of the $U(1)$ gauge coupling $e$. The four-dimensional Lorentz invariance requires

$$
A_{m}=0, \quad \hat{A}_{m}=0, \quad \lambda_{1}=0, \quad \lambda_{2}=0, \quad \psi_{i}=0, \quad \tilde{\psi}^{i}=0 .
$$


We also demand that all the remaining dynamical fields $\phi, \hat{\phi}, q_{i}, \tilde{q}^{i}$ and the auxiliary fields $F_{\Phi}, \hat{F}_{\Phi}, D, \hat{D}, F_{i}, \tilde{F}^{i}$ depend on only extra coordinates $x^{5}, x^{6}$. Then the bosonic part of the Lagrangian reduces to

$$
\begin{aligned}
\mathcal{L}_{\text {boson }}^{(5,6)=} & \frac{1}{g^{2}} \operatorname{Tr}\left[-\frac{1}{2}\left(\hat{F}_{56}\right)^{2}+\frac{1}{2} \hat{D}^{2}+\left|\hat{F}_{\Phi}\right|^{2}\right]+\frac{1}{e^{2}}\left[-\frac{1}{2}\left(F_{56}\right)^{2}+\frac{1}{2} D^{2}+\left|F_{\Phi}\right|^{2}\right] \\
& -\overline{\mathcal{D}} q^{\dagger i} \mathcal{D} q_{i}+\frac{1}{2} q^{\dagger i}\left(F_{56}+\hat{F}_{56}\right) q_{i}+F^{\dagger i} F_{i}-\frac{1}{2} q^{\dagger i}(D+\hat{D}) q_{i} \\
& -\mathcal{D} \tilde{q}^{i} \overline{\mathcal{D}} \tilde{q}^{\dagger}{ }_{i}-\frac{1}{2} \tilde{q}^{i}\left(F_{56}+\hat{F}_{56}\right) \tilde{q}_{i}^{\dagger}+\tilde{F}^{i} \tilde{F}_{i}^{\dagger}+\frac{1}{2} \tilde{q}^{i}(D+\hat{D}) \tilde{q}_{i}^{\dagger} \\
& -\frac{1}{\sqrt{2}} \tilde{q}^{i}\left(F_{\Phi}+\hat{F}_{\Phi}\right) q_{i}+\frac{v^{2}}{2 \sqrt{2}} F_{\Phi}-\frac{1}{\sqrt{2}} q^{\dagger i}\left(\bar{F}_{\Phi}+\hat{F}_{\Phi}^{\dagger}\right) \tilde{q}_{i}^{\dagger}+\frac{v^{2}}{2 \sqrt{2}} \bar{F}_{\Phi} .
\end{aligned}
$$

In order to obtain vortex solutions, we further put an Ansatz

$$
q_{i}^{a}=\tilde{q}_{i}^{\dagger a} \rightarrow \frac{1}{\sqrt{2}} q_{i}^{a}
$$

reexpressing these using the same character $q^{a}{ }_{i}$. Eliminating the auxiliary fields by their algebraic equations of motion, the above Lagrangian becomes

$$
\begin{aligned}
\mathcal{L}_{\text {vort }} & =-\frac{1}{2 g^{2}} \sum_{A=1}^{N^{2}-1}\left(F_{56}^{A}\right)^{2}-\frac{1}{2 e^{2}}\left(F_{56}\right)^{2}-\frac{1}{2} \overline{\mathcal{D}} q^{\dagger i} \mathcal{D} q_{i}-\frac{1}{2} \mathcal{D} q^{\dagger i} \overline{\mathcal{D}} q_{i}-V_{\text {vort }}, \\
V_{\text {vort }} & =\frac{g^{2}}{8} \sum_{A=1}^{N^{2}-1}\left(q^{\dagger i} T^{A} q_{i}\right)^{2}+\frac{e^{2}}{8}\left(q^{\dagger i} q_{i}-v^{2}\right)^{2} .
\end{aligned}
$$

The stability of the vortices in this model is ensured by the nontrivial topological winding number (2.24) arising from the color-flavor locking vacuum

$$
\left|q^{a}{ }_{i}\right|=\frac{v}{\sqrt{N}} \delta^{a}{ }_{i}
$$

The tension of a vortex solution is given by

$$
\begin{aligned}
& T_{\mathrm{NA}}=\int d^{2} x {\left[\frac{1}{2 g^{2}} \sum_{A=1}^{N^{2}-1}\left(F_{56}^{A}\right)^{2}+\frac{1}{2 e^{2}}\left(F_{56}\right)^{2}+\frac{1}{2} \overline{\mathcal{D}} q^{\dagger i} \mathcal{D} q_{i}+\frac{1}{2} \mathcal{D} q^{\dagger i} \overline{\mathcal{D}} q_{i}+V\right] } \\
&=\int d^{2} x\left[\frac{1}{2} \sum_{A=1}^{N^{2}-1}\left(\frac{1}{g} F_{56}^{A}-\frac{g}{2} q^{\dagger i} T^{A} q_{i}\right)^{2}+\frac{1}{2}\left\{\frac{1}{e} F_{56}-\frac{e}{2}\left(q^{\dagger i} q_{i}-v^{2}\right)\right\}^{2}\right. \\
&\left.+\overline{\mathcal{D}} q^{\dagger i} \mathcal{D} q_{i}-\frac{v^{2}}{2} F_{56}\right]
\end{aligned}
$$

with $d^{2} x \equiv d x^{5} d x^{6}=2 i d z d z^{*}$, where we have used

$$
\mathcal{D} q^{\dagger i} \overline{\mathcal{D}} q_{i}=\overline{\mathcal{D}} q^{\dagger i} \mathcal{D} q_{i}-q^{\dagger i}\left(F_{56}+\hat{F}_{56}\right) q_{i}
$$


It is bounded from below by

$$
T_{\mathrm{NA}} \geq-\frac{v^{2}}{2} \int d^{2} x F_{56}
$$

This inequality is saturated when the following BPS equations are satisfied [22, 21] :

$$
\begin{aligned}
F_{56}^{A} & =\frac{g^{2}}{2} q^{\dagger i} T^{A} q_{i}, \\
F_{56} & =\frac{e^{2}}{2}\left(q^{\dagger i} q_{i}-v^{2}\right), \\
\mathcal{D} q_{i} & =0 .
\end{aligned}
$$

These are natural generalizations of the BPS equations for the $U(1)$ vortices [16, 17].

The $U(1)$ vortices are solutions of the Abelian-Higgs model which is obtained from (2.14) by retaining only the $U(1)$ vector multiplet $(V, \Phi)$ and a single charged hypermultiplet $q$. The BPS equations in this $U(1)$ case are of the form:

$$
F_{56}=\frac{e^{2}}{2}\left(|q|^{2}-v^{2}\right), \quad \mathcal{D} q=0 .
$$

If the topological winding number $\pi_{1}(U(1))$ in Eq. (2.24) is $k$, we assume the vortex Ansatz with profile functions $\varphi$ and $f$ as a function of $r$

$$
q=\mathrm{e}^{-i k \theta} \varphi(r), \quad A_{\mu}=2 \varepsilon_{\mu \nu} \frac{x^{\nu}}{r^{2}}(k-f(r)),
$$

where $(r, \theta)$ are polar coordinates on the $x_{5}-x_{6}$ plane, $\mu, \nu$ run from 5 to 6 and the antisymmetric tensor is defined as $\varepsilon_{56}=1$. Then the above BPS equations reduce to

$$
2 \frac{f^{\prime}}{r}=\frac{e^{2}}{2}\left(\varphi^{2}-v^{2}\right), \quad r \varphi^{\prime}=f \varphi
$$

The profile functions have to satisfy the following boundary conditions

$$
\begin{gathered}
f(\infty)=0, \quad \varphi(\infty)=v, \quad f(0)=k, \\
\varphi(0)=0, \quad \text { if } \quad k \neq 0 .
\end{gathered}
$$

The tension of the $U(1)$ BPS vortex with the winding number $k$ is given by [16, 17]

$$
T_{U(1)}=-\frac{v^{2}}{2} \int d^{2} x F_{56}=-\frac{v^{2}}{2} \int d^{2} x 2 \frac{f^{\prime}}{r}=-2 \pi v^{2}[f]_{0}^{\infty}=2 \pi k v^{2} .
$$

We will call the $U(1)$ vortex with the unit winding number as ANO vortex whose tension is given by

$$
T_{\mathrm{ANO}}=2 \pi v^{2}
$$


The existence of the solution for the $U(1)$ BPS equations (3.15) with the winding number $k$ has been demonstrated and its power series expansion has been studied [18], although no explicit analytic solution has been obtained so far.

Let us turn our attention to the non-Abelian vortices by solving Eqs. (3.10)-(3.12). We first reexpress them in a matrix form defined by [21]

$$
\check{A}_{\mu} \equiv A_{\mu} \mathbf{1}_{N}+\hat{A}_{\mu}, \quad \check{F}_{\mu \nu} \equiv F_{\mu \nu} \mathbf{1}_{N}+\hat{F}_{\mu \nu} .
$$

Then Eqs. (3.10) and (3.11) are combined into the following matrix equation:

$$
\check{F}_{56}=\frac{g^{2}}{2}\left(q_{i} q^{\dagger i}-\frac{v^{2}}{N} \mathbf{1}_{N}\right)+\left(\frac{e^{2}}{2}-\frac{g^{2}}{2 N}\right) \operatorname{Tr}\left(q_{i} q^{\dagger i}-\frac{v^{2}}{N} \mathbf{1}_{N}\right) \mathbf{1}_{N} .
$$

Let us first consider the case of general gauge couplings $e$ and $g$. A natural vortex Ansatz corresponding to a highly symmetric point in the internal space and in the extra dimension, namely the cylindrically symmetric Ansatz with a common origin for all the vortices, is given by

$$
\begin{aligned}
& q=\frac{1}{\sqrt{N}}\left(\begin{array}{llll}
\mathrm{e}^{-i \ell_{1} \theta} \varphi_{1}(r) & & & \\
& \mathrm{e}^{-i \ell_{2} \theta} \varphi_{2}(r) & & \\
& & \ddots & \\
& & & \mathrm{e}^{-i \ell_{N} \theta} \varphi_{N}(r)
\end{array}\right), \\
& \check{A}_{\mu}=2 \varepsilon_{\mu \nu} \frac{x^{\nu}}{r^{2}}\left(\begin{array}{llll}
\ell_{1}-f_{1}(r) & & & \\
& \ell_{2}-f_{2}(r) & & \\
& & \ddots & \\
& & & \ell_{N}-f_{N}(r)
\end{array}\right) \text {, }
\end{aligned}
$$

where $\ell_{i}(i=1,2, \cdots, N)$ are integers. Inserting this Ansatz into the above matrix equation (3.21), one finds [22]

$$
2 \frac{f_{i}^{\prime}}{r}=\frac{g^{2}}{2 N}\left(\varphi_{i}^{2}-v^{2}\right)+\left(\frac{e^{2}}{2}-\frac{g^{2}}{2 N}\right)\left(\frac{1}{N} \sum_{j=1}^{N} \varphi_{j}^{2}-v^{2}\right) .
$$

The remaining equation (3.12) gives

$$
r \varphi_{i}^{\prime}=f_{i} \varphi_{i}
$$

Comparing these equations with Eqs. (3.15) for the $U(1)$ vortex, we recognize that the above Ansatz for the non-Abelian vortex is a natural extension of the Ansatz (3.14). The 
cylindrically symmetric profile functions $\left(f_{i}, \varphi_{i}\right)$ should satisfy the following boundary conditions:

$$
f_{i}(0)=\ell_{i}, \quad \varphi_{i}(\infty)=v, \quad f_{i}(\infty)=0 .
$$

Moreover, the BPS equation (3.25) and the boundary conditions (3.26) require the boundary condition for $\varphi_{i}(0)$

$$
\varphi_{i}(0)=0, \quad \text { if } \quad \ell_{i} \neq 0 .
$$

Notice that Eq. (3.9) implies that the tension of the BPS vortex is determined only by the $U(1)$ part of the gauge group. In fact, the tension of the non-Abelian vortices is given by

$$
T_{\mathrm{NA}}=-\frac{v^{2}}{2} \int d^{2} x F_{56}=-\frac{v^{2}}{2} \int d^{2} x \frac{2}{N r} \sum_{i=1}^{N} f_{i}^{\prime}=\frac{2 \pi v^{2}}{N} \sum_{i=1}^{N} \ell_{i} .
$$

This implies that the winding number $k \in \pi_{1}(U(1))$ defined in (2.24) is the sum of $\ell_{i}$ :

$$
k=\sum_{i=1}^{N} \ell_{i} .
$$

Therefore the non-Abelian vortices with the topological winding number $k$ gives the tension which amounts to $1 / N$ of the $k$ ANO vortices

$$
T_{\mathrm{NA}}=\frac{k}{N} T_{\mathrm{ANO}} .
$$

The minimal vortex solutions with $\ell_{i}=1, \ell_{j}=0(j \neq i, 1 \leq j \leq N)$ have tension whose value is $1 / N$ of that of the ANO vortex in Eq. (3.19).

When we have no particular relation between two gauge couplings $e$ and $g$, Eq. (3.24) shows that the profile functions $f_{i}$ and $\varphi_{i}$ satisfy coupled differential equations whose solutions are generally different from those for the $U(1)$ vortices. Solutions have been obtained numerically in the case of $N=2$ in Ref. [22]. They give both profile functions $\varphi_{1}$ and $\varphi_{2}$ which are nontrivial and are different from the $U(1)$ vortex solutions [22].

In the case of $\ell_{1}=\ell_{2}=\cdots=\ell_{N}=\ell$, however, the cylindrically symmetric boundary conditions (3.26) and (3.27), and the BPS equations (3.24) and (3.25) allow a solution for the profile functions which become identical to the vortex solutions for the $U(1)$ BPS equations (3.15). To see this, we note that the boundary conditions (3.26) and (3.27) in this case allow the following Ansatz

$$
\varphi_{i}(r)=\varphi(r), \quad f_{i}(r)=f(r), \quad(i=1,2, \cdots, N),
$$


in Eqs. (3.24) and (3.25). Then we find that the BPS equations for $f$ and $\varphi$ become completely identical to those of the $U(1)$ vortex [22]:

$$
2 \frac{f^{\prime}}{r}=\frac{e^{2}}{2}\left(\varphi^{2}-v^{2}\right), \quad r \varphi^{\prime}=f \varphi
$$

In contrast to the minimal vortices, this configuration with $\ell_{1}=\ell_{2}=\cdots=\ell_{N}=\ell$ winds $\ell$ full turns around the $U(1)$ part without going through the $S U(N)$ part. Notice that the solution is valid for generic values of $U(1)$ gauge coupling $e$ and that the tension of this $\ell_{1}=\ell_{2}=\cdots=\ell_{N}=\ell$ vortex is the same as that for the $\ell$ ANO vortices as shown in Eqs. (3.18) and (3.19).

Next let us consider the special case where the gauge couplings $g$ and $e$ satisfy a particular relation 21 ]

$$
g^{2}=N e^{2}
$$

In this case the second term of the right hand side of Eq. (3.21) vanishes. This implies that the diagonal entries have no interaction with each other, if we stick to the configurations with vanishing off-diagonal entries. We denote diagonal entries of such a configuration as $q_{\star}^{(i)}$ and $F_{56 \star}^{(i)}(i=1, \cdots, k)$

$$
\left(\check{F}_{56}\right)_{b}^{a}=\left(\begin{array}{cccc}
F_{56 \star}^{(1)} & & & \\
& F_{56 \star}^{(2)} & & \\
& & \ddots & \\
& & & F_{56 \star}^{(n)}
\end{array}\right), \quad q^{a}{ }_{i}=\frac{1}{\sqrt{N}}\left(\begin{array}{cccc}
q_{\star}^{(1)} & & & \\
& q_{\star}^{(2)} & & \\
& & \ddots & \\
& & q_{\star}^{(n)}
\end{array}\right) .
$$

Eqs. (3.12) and (3.21) give the $N$ decoupled sets of the BPS equations for this configuration

$$
F_{56 \star}^{(i)}=\frac{e^{2}}{2}\left(\left|q_{\star}^{(i)}\right|^{2}-v^{2}\right), \quad \mathcal{D} q_{\star}^{(i)}=0, \quad(i=1, \cdots, N) .
$$

which turn out to be identical to the BPS eqs. (3.13) for the $U(1)$ theory. The solution for each component is given by the following vortex Ansatz

$$
q_{\star}^{(i)}=\mathrm{e}^{-i \ell_{i} \theta_{i}} \varphi_{i}\left(r_{i}\right), \quad A_{\star \mu}^{(i)}=2 \varepsilon_{\mu \nu} \frac{x^{\nu}}{r_{i}^{2}}\left(\ell_{i}-f_{i}\left(r_{i}\right)\right),
$$

where $\left(r_{i}, \theta_{i}\right)$ are polar coordinates on the $x_{5}-x_{6}$ plane whose origins are generally different from each other. Thus we obtain $N$ sets of BPS equations for the $U(1)$ vortices:

$$
2 \frac{f_{i}^{\prime}}{r_{i}}=\frac{e^{2}}{2}\left(\varphi_{i}^{2}-v^{2}\right), \quad r_{i} \varphi_{i}^{\prime}=f_{i} \varphi_{i}, \quad(i=1,2, \cdots, N)
$$


We should stress that the cylindrical symmetry with the common origin for all $i=$ $1, \cdots, N$ is not needed, since diagonal entries are decoupled unlike the case of general gauge couplings $e$ and $g$. The boundary conditions are given by

$$
\left.f_{i}\right|_{r_{i}=0}=\ell_{i},\left.\quad \varphi_{i}\right|_{r_{i}=0}=0,\left.\quad f_{i}\right|_{r_{i}=\infty}=0,\left.\quad \varphi_{i}\right|_{r_{i}=\infty}=v
$$

In this case the minimal solution with $\ell_{i}=1, \ell_{j}=0, j \neq i(1 \leq j \leq N)$ is simply given by $\varphi_{j \neq i}=v$ and $f_{j \neq i}=0$, and the nontrivial profile functions $\varphi_{i}$ and $f_{i}$ are exactly the same as those for the ANO vortex. Taking the case of $\ell_{1}=1$ as an example, the Ansatz for a minimal vortex reduces to

$$
q=\frac{1}{\sqrt{N}}\left(\begin{array}{cccc}
\mathrm{e}^{-i \theta} \varphi_{1} & & & \\
& v & & \\
& & \ddots & \\
& & & v
\end{array}\right), \check{A}_{\mu}=2 \varepsilon_{\mu \nu} \frac{x^{\nu}}{r^{2}}\left(\begin{array}{cccc}
1-f_{1} & & & \\
& 0 & & \\
& & \ddots & \\
& & 0
\end{array}\right) .
$$

This type of solutions has been considered in Ref. 21] for the case of three spacetime dimensions.

\section{Effective Field Theory on Vortices}

In this section we discuss the effective field theory on vortices restricting to the case of $g^{2}=N e^{2}$ in Eq. (3.33). When Eq. (3.33) does not hold, the effective theory is expected to be deformed. We will return to this problem in Sec. 5 .

In the first subsection, we discuss the effective field theory on non-Abelian vortices in a purely field theoretical context, by starting from a single vortex or noninteracting multivortex solutions. It works well for a single vortex but not for multi-vortices because we do not find correct number of bosons needed for the moduli space dimension. In the second subsection, we discuss the moduli space for multi-vortices inspired by branes which are proposed by Hanany and Tong [21, and find that their effective theory contains a number of extra massless bosons, which are called quasi-Nambu-Goldstone bosons. Combined with the genuine Nambu-Goldstone bosons, we obtain all the massless bosons, exhausting the correct dimensions of the moduli space. As shown in 21], the Hanany-Tong metric does not coincide with the Manton metric even for two vortices in the $U(1)$ gauge theory. Hence we do not expect that it is a correct metric describing the scattering of vortices but that it has correct dimensions, topology and symmetry. We discuss this problem in the next section. Moreover the brane construction in [21] was given for three-dimensional 
theory and later it was generalized to four dimensions in 27] taking a T-dual. We simply use the same Lagrangian for six dimensions because the structure of the moduli space is independent of dimensions at least classically.

\subsection{Orientation Moduli for Vortices}

Dynamics of vortices is described by the effective field theory of massless fields on the vortices. The Manton method [6] or the mode expansion method can be used to construct the effective field theory, which is in general a nonlinear sigma model with the moduli space of vortices as its target manifold. Since we do not have explicit solutions even for the ANO vortex of the $U(1)$ gauge theory, it is difficult to integrate over the extra dimensions. However in the case of a single non-Abelian vortex, the moduli space metric is determined by symmetry only with no need of the explicit solution.

First, let us consider the effective field theory on a single non-Abelian vortex. The BPS equations (3.10)-(3.12) are invariant under a global symmetry $S U(N)_{\mathrm{G}+\mathrm{F}}$ given in (2.26). Namely, when a set of $\check{F}_{56}$ and $q$ is a solution, then the following transformation keeps it as a solution of the BPS equations

$$
\check{F}_{56} \rightarrow U_{\mathrm{G}} \check{F}_{56} U_{\mathrm{G}}^{-1}, \quad q \rightarrow U_{\mathrm{G}} q U_{\mathrm{F}} \quad\left(U_{\mathrm{G}} \in S U(N)_{\mathrm{G}}, \quad U_{\mathrm{F}}=U_{\mathrm{G}}^{-1} \in S U(N)_{\mathrm{F}}\right)
$$

Since the configuration at the spatial infinity in the $x^{5}-x^{6}$ plane is fixed under this transformation, the corresponding zero modes should be normalizable physical modes. This fact implies that the moduli space metric admits an isometry $S U(N)_{\mathrm{G}+\mathrm{F}}$. On the other hand, our Ansatz (3.39) is invariant under the subgroup $H \equiv S U(N-1) \times U(1)$ of $S U(N)_{\mathrm{G}+\mathrm{F}}$. So $S U(N)_{\mathrm{G}+\mathrm{F}}$ is spontaneously broken down to its subgroup $H$ by the vortex configuration (3.39), and $H$ is the isotropy group of the moduli space metric. We thus have observed that the moduli space for a single non-Abelian vortex, denoted by $\mathcal{M}_{1, N}$, can be written as 21,22

$$
\mathcal{M}_{1, N}=\mathbf{C} \times \frac{S U(N)_{\mathrm{G}+\mathrm{F}}}{S U(N-1) \times U(1)} \simeq \mathbf{C} \times \mathbf{C} P^{N-1} .
$$

The first factor $\mathbf{C}$ corresponds to the Nambu-Goldstone degree of freedom for the vortex position $z_{0}$ in the $x^{5}-x^{6}$ plane which exists for Abelian cases also. Whereas the second part is parametrized by Nambu-Goldstone bosons arising from spontaneously broken internal symmetry. ${ }^{3}$ This is a characteristic feature in non-Abelian cases. The complex dimension

\footnotetext{
${ }^{3}$ In eight SUSY theories, domain walls are known to be accompanied with the Nambu-Goldstone bosons for broken internal $U(1)$ symmetry [15].
} 
of the above metric is $N$ in agreement with the index theorem calculated by Hanany and Tong [21] generalizing the Abelian case [29, and therefore we do not have any other massless modes.

The moduli space of a single vortex has been completely determined by symmetry only. In the case of multi-vortices, we need more considerations as shown below. Let us denote the moduli space of $k$ vortices by $\mathcal{M}_{k, N}$ and consider the case of $k \leq N$ :

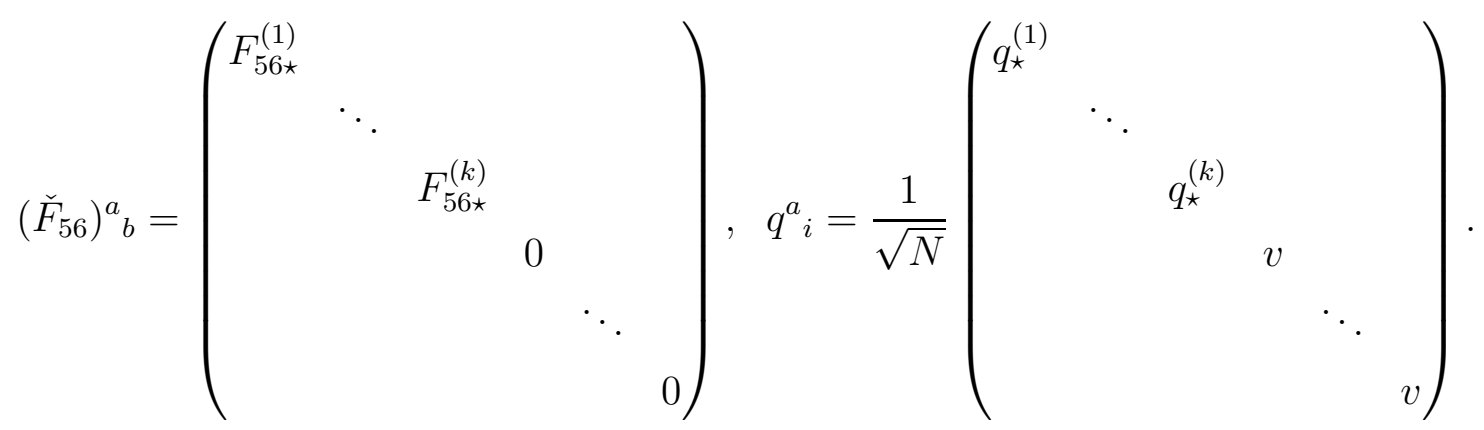

Here, $q_{\star}^{(I)}$ and $F_{56 \star}^{(I)}(I=1, \cdots, k)$ are solutions of the decoupled $U(1)$ vortices :

$$
q_{\star}^{(I)}=q_{\star}\left(z-z_{I}, z^{*}-z_{I}^{*}\right), \quad F_{56 \star}^{(I)}=F_{56 \star}\left(z-z_{I}, z^{*}-z_{I}^{*}\right), \quad I=1, \cdots, k,
$$

where $z_{I}$ are the positions of the $I$-th vortex. Since these $k$ vortices do not interact with each other in this particular Ansatz of diagonal embedding, which corresponds to a particular sector (submanifold) of the full moduli space. Let us note that the above solution of non-Abelian vortices is exact irrespective of the position of the vortices $z_{I}$. Therefore these moduli $z_{I}$ are exact translational moduli of the $k$-vortex solution.

The symmetry $S U(N)_{\mathrm{G}+\mathrm{F}}$ is spontaneously broken by the configuration (4.3) down into $S U(N-k) \times U(1)^{k}$ provided $z_{I} \neq z_{J}$ for $I \neq J$. Hence the Nambu-Goldstone bosons for this breaking appear and parametrize the coset $S U(N) /\left[S U(N-k) \times U(1)^{k}\right]$ inside the moduli space of $k$-vortices $\mathcal{M}_{k, N}$. We also have moduli $z_{I}$ for translation of vortices. Therefore the moduli space certainly contains

$$
\mathrm{C}^{k} \times \frac{S U(N)_{\mathrm{G}+\mathrm{F}}}{S U(N-k) \times U(1)^{k}} \quad\left(\subset \mathcal{M}_{k, N}\right),
$$

as a submanifold. This does not coincides with the whole moduli space of $k$-vortices since the complex dimension of (4.5) is $k N-\frac{1}{2} k(k-1)$, which is less than $\operatorname{dim}_{\mathbf{C}} \mathcal{M}_{k, N}=k N$ calculated using the index theorem [21]. This comes from the fact that we are considering a particular Ansatz (4.3) with too small number of parameters. We thus have missed $\frac{k(k-1)}{2}$ additional complex moduli parameters, which will turn out to be the so-called quasiNambu-Goldstone modes when we consider the low-energy effective Lagrangian [8]-[14] in the next subsection. 
In the case $z_{I}=z_{J}$ for a set of $I, J$ with $I \neq J$ the unbroken symmetry is enhanced to $S U(N-k) \times U(2) \times U(1)^{k-2}$, and we find the moduli space includes

$$
\mathrm{C}^{k} \times \frac{S U(N)_{\mathrm{G}+\mathrm{F}}}{S U(N-k) \times U(2) \times U(1)^{k-2}} \quad\left(\subset \mathcal{M}_{k, N}\right)
$$

as a submanifold with the complex dimension $k N-\frac{1}{2} k(k-1)-1$. Similarly, depending on how many translational moduli $z_{I}$ coincide, several different coset manifolds of NambuGoldstone modes are embedded into the moduli space. Let us consider the case of $n_{a}$ $\left(a=1, \cdots, m ; \sum_{a=1}^{m} n_{a} \leq k\right)$ of them coincide like

$$
\left(z_{1}, \cdots, z_{k}\right)=(\underbrace{z_{(1)}, \cdots, z_{(1)}}_{n_{1}} ; \cdots ; \underbrace{z_{(m)}, \cdots, z_{(m)}}_{n_{m}} ; z_{(m+1)} ; z_{(m+2)} ; \cdots ; z_{\left(k-\sum_{a=1}^{m} n_{a}+m\right)}) .
$$

Then the coset manifolds of Nambu-Goldstone modes

$$
\mathrm{C}^{k} \times \frac{S U(N)_{\mathrm{G}+\mathrm{F}}}{S U(N-k) \times \prod_{a} S U\left(n_{a}\right) \times U(1)^{k+m-\sum_{a} n_{a}}} \quad\left(\subset \mathcal{M}_{k, N}\right)
$$

with the complex dimension $N k-\frac{1}{2} k(k-1)-\sum_{a=1}^{m} \frac{1}{2} n_{a}\left(n_{a}-1\right)$, is embedded into the full moduli space.

In either case we did not find enough number of massless bosons parametrizing the full moduli space. Instead we have several coset spaces with various dimensions parametrized by Nambu-Goldstone bosons for several breaking patterns of internal symmetry $S U(N)_{\mathrm{G}+\mathrm{F}}$. This is actually a feature for symmetry breaking in SUSY (gauge) theories with quasiNambu-Goldstone bosons [12, 13. As discussed in the next subsection, such a situation can be completely described by the moduli space theory formulated by a SUSY gauge theory (or a Kähler quotient) which was proposed by Hanany and Tong (using the brane configuration in the string theory) [21].

Before closing this subsection, we make a comment. When we embed the solutions of the $U(1)$ vortices into diagonal entries in $\left(\check{F}_{56}\right)^{a}{ }_{b}$ and $q^{a}{ }_{i}$ in Eq. (4.3), these vortices do not interact. However, if we would like to consider an Ansatz of vortices placed in off-diagonal entries unlike Eq. (4.3), it is no longer an exact solution of the BPS equations. This fact implies that these vortices are interacting. These vortices become exact solutions only in the limit of infinite separation between vortices. We cannot avoid this situation in the case of large numbers of vortices $k \geq N$.

\subsection{Symmetry Structure in the Vortex Moduli Space}

Inspired by brane configurations, an effective theory on $k$-vortices in the $U(N)$ gauge theory with $N$ hypermultiplets was given by Hanany and Tong [21] in the form of a 
Kähler quotient. It is described by $D=4, \mathcal{N}=1 \mathrm{SUSY}$ (with four supercharges) $U(k)$ gauge theory with auxiliary vector superfields of a $k$ by $k$ matrix $V\left(x^{m}, \theta, \bar{\theta}\right)$ (without kinetic term) and matter chiral superfields of a $k$ by $N$ matrix $\varphi\left(x^{m}, \theta, \bar{\theta}\right)$ and a $k$ by $k$ adjoint matrix $Z\left(x^{m}, \theta, \bar{\theta}\right)$. Their $U(k)$ supergauge transformation laws are given by

$$
\begin{aligned}
& e^{V} \rightarrow e^{V^{\prime}}=e^{-\Lambda^{\dagger}} e^{V} e^{-\Lambda}, \\
& Z \rightarrow Z^{\prime}=e^{\Lambda} Z e^{-\Lambda}, \\
& \varphi \rightarrow \varphi^{\prime}=e^{\Lambda} \varphi,
\end{aligned}
$$

with $\Lambda\left(x^{m}, \theta, \bar{\theta}\right)$ a $k$ by $k$ matrix chiral superfield for a gauge parameter. Note that the gauge symmetry is complexified to $U(k)^{\mathbf{C}}=G L(k, \mathbf{C})$. The effective Lagrangian proposed by Hanany and Tong is

$$
\mathcal{L}_{\mathrm{HT}}=\int d^{4} \theta\left[\operatorname{tr}\left(\varphi \varphi^{\dagger} e^{V}\right)+\operatorname{tr}\left(Z^{\dagger} e^{V} Z e^{-V}\right)-c \operatorname{tr} V\right]
$$

with $c$ a Fayet-Iliopoulos parameter related to the gauge coupling $g$ of the original gauge theory by $c=2 \pi / g^{2}$. This theory has a global symmetry $S U(N)$ originated from $S U(N)_{\mathrm{G}+\mathrm{F}}$ :

$$
\varphi \rightarrow \varphi^{\prime}=\varphi U,
$$

with $U$ the $S U(N)$ unitary matrix in the fundamental representation. The Higgs branch of this theory is an $U(k)$ Kähler quotient $\mathcal{M}_{k, N}=\{Z, \varphi\} / / U(k)=\{Z, \varphi\} / U(k)^{\mathbf{C}}$ with $\operatorname{dim}_{\mathrm{C}} \mathcal{M}_{k, N}=k N$, and the theory is described by a nonlinear sigma model with $\mathcal{M}_{k, N}$ as the target space. Since we have chosen the minimal kinetic term as a first guess, we do not expect that the metric $\mathcal{M}_{k, N}$ coincides exactly with the Manton metric, although their dimension, topology and symmetry should coincide.

Here we investigate the geometric structure of the moduli space. The Wess-Zumino gauge makes physical content transparent, but breaks $U(k)^{\mathbf{C}}$ to $U(k)$. In the following we do not take the Wess-Zumino gauge, and use the full complex gauge symmetry for fixing superfields. First, in the $k=1$ case of the single vortex, the Lagrangian (4.10) is the Kähler quotient formulation for $\mathbf{C} P^{N-1}$ [37, 33]. Eliminating $V$ by its equation of motion, $\varphi \varphi^{\dagger} e^{V}-c=0$, we obtain the Kähler potential for the Fubini-Study metric on $\mathbf{C} P^{N-1}, K=c \log \left(1+\hat{\varphi} \hat{\varphi}^{\dagger}\right)$ with a gauge fixing $\varphi=(1, \hat{\varphi})$. In this case the moduli space agrees with the one parametrized by the Nambu-Goldstone bosons only, as discussed in the last subsection.

Next we investigate the moduli space geometry in the case of $1<k \leq N$. In this case, it is difficult to eliminate $V$ in the superfield formalism. Instead, we fix the gauge without 
eliminating $V$. Although we cannot get the Kähler potential in terms of independent fields we can identify fields as Nambu-Goldstone or quasi-Nambu-Goldstone bosons. We will see that there exist some quasi-Nambu-Goldstone bosons which are not related to the spontaneously broken global symmetry. First of all, using the gauge symmetry (4.9), $Z$ can be diagonalized as

$$
Z_{\text {fixed }}=\left(\begin{array}{ccc}
z_{1} & & \mathbf{0} \\
& \ddots & \\
\mathbf{0} & & z_{k}
\end{array}\right)
$$

where each $z_{I}(I=1, \cdots, k)$ is interpreted as the position of each vortex. The complex gauge group $U(k)^{\mathbf{C}}$ is broken down to its subgroup $\left\{U(1)^{\mathbf{C}}\right\}^{k}$ by the VEVs of (4.12), provided $z_{I} \neq z_{J}$ for $I \neq J$. Using this unbroken gauge symmetry, $k$ components in $\varphi$ can be fixed to unity (we take them to be diagonal entries) :

$$
\varphi_{\text {fixed }}=\left(\begin{array}{ccccc}
1 & D_{11} & \cdots & \cdots & D_{1, k-1} \\
C_{11} & 1 & \ddots & & D_{2, k-1} \\
\vdots & \ddots & \ddots & \ddots & \vdots \\
C_{1, k-2} & \cdots & \ddots & 1 & D_{k-1, k-1} \\
C_{1, k-1} & C_{2, k-1} & \cdots & C_{k-1, k-1} & 1
\end{array}\right) E_{k \times(N-k)}
$$

with $C_{I J}(I \leq J=1, \cdots, k-1), D_{I J}(I \geq J=1, \cdots, k-1)$ and $E$ chiral superfields. Note that $\varphi$ has no independent degrees of freedom in the Abelian case $N=1$; it parametrizes the orientation of the vortices in the internal space in the non-Abelian case.

After fixing the gauge, we take the VEVs of these dynamical fields. Fluctuations of these fields from those VEVs are identified with propagating fields. In our case they are interpreted as Nambu-Goldstone or quasi-Nambu-Goldstone bosons. Depending on VEVs, the numbers of these bosons can vary with their total number unchanged [12, 13. VEVs transformed by the global symmetry (4.11) are equivalent to each other, and so physics for instance the identification of the Nambu-Goldstone or quasi-Nambu-Goldstone bosons is unchanged. If we compare two VEVs which are not related by the global symmetry, physics generically changes: unbroken symmetry can change, the number of Nambu-Goldstone and quasi-Nambu-Goldstone can vary or, even in the case of the same unbroken symmetry, decay constants of Nambu-Goldstone bosons are different in general.

Therefore when we take the VEVs of (4.12) and (4.13), to identify Nambu-Goldstone or quasi-Nambu-Goldstone bosons, we can use the global $S U(N)$ transformations (4.11). Since these $S U(N)$ transformations generically break the gauge fixing conditions (4.13) 
for $\left\{U(1)^{\mathbf{C}}\right\}^{k}$, we need to perform supplementary $\left\{U(1)^{\mathbf{C}}\right\}^{k}$ transformations to pull back to the gauge fixing hypersurface (4.13). The (representative of) VEVs of $\varphi$ are given by

$$
\langle\varphi\rangle=\left(\begin{array}{ccccc|c}
1 & 0 & \cdots & \cdots & 0 & \\
* & 1 & \ddots & & \vdots & \\
\vdots & \ddots & \ddots & \ddots & \vdots & 0_{k \times(N-k)} \\
* & \cdots & \ddots & 1 & 0 & \\
* & * & \cdots & * & 1
\end{array}\right) .
$$

$\frac{1}{2} k(k-1)$ lower left components denoted by $*$ are not fixed by any symmetry and so are identified with the VEVs of quasi-Nambu-Goldstone bosons. When we change these parameters, the homogeneous space representing the Nambu-Goldstone bosons is gradually deformed. When there are generically $n$ parameters to deform a homogeneous space, it is called cohomogeneity $k$. By counting *'s in (4.14) and $z_{I}$ in (4.12) we find that the moduli manifold is of cohomogeneity $2\left[\frac{1}{2} k(k-1)+k\right]=k(k+1)$. Therefore $C_{I J}$ in the decomposition (4.13) can be identified as the quasi-Nambu-Goldstone bosons, which are the parameters of the solution without accompanying spontaneously broken global symmetries.

Let us consider the case where all the $*$ 's in (4.14) vanish. Then the total symmetry $U(k)_{\mathrm{g}} \times S U(N)_{\mathrm{f}}$ is broken down to a global symmetry $S U(N-k)_{\mathrm{f}} \times U(1)_{\mathrm{g}+\mathrm{f}}^{k}$ by VEVs (4.12) and (4.14) with no unbroken gauge symmetry (the suffixes $g$ and $f$ represent the gauge and flavor symmetries in the vortex world-volume theory (4.10) ). Here $U(1)_{\mathrm{g}+\mathrm{f}}^{k}$ is given by the $I$-th rotation $\left(I=1, \cdots, k\right.$ of unbroken $U(1)^{k}$ gauge symmetry and an $S U(N)$ generator

$$
\operatorname{diag} .(0, \cdots, 0, \underbrace{N-k}_{I \text {-th }}, 0, \cdots, 0, \underbrace{-1, \cdots,-1}_{N-k})
$$

with the opposite angles. By this breaking there appear Nambu-Goldstone bosons parametrizing $S U(N) / S U(N-k) \times U(1)^{k}$. Comparing (4.13) and (4.14) these Nambu-Goldstone bosons sit in $D$ and $E$ in (4.13). The quasi-Nambu-Goldstone bosons sit in real $2 k$ components $z_{I}$ in Eq. (4.12) and $k(k-1)$ components $C_{I J}$ in Eq. (4.13). They parametrize non-compact directions of the moduli space $\mathcal{M}_{k, N}$ while the Nambu-Goldstone bosons parametrize compact directions. Therefore $\mathcal{M}_{k, N}$ can be locally written $\operatorname{as}^{4}$

$$
\mathcal{M}_{k, N}=\mathbf{C}^{k} \times \mathbf{C}^{\frac{1}{2} k(k-1)} \times \frac{S U(N)_{\mathrm{G}+\mathrm{F}}}{S U(N-k) \times U(1)^{k}} .
$$

\footnotetext{
${ }^{4}$ Note that the expression by the direct product is not rigorous in the mathematical sense because we decomposed it in the level of the algebra but not of the group.
} 
One should note that the index $G+F$ represents the diagonal symmetry group for the original theory instead of the vortex theory. Comparing this with (4.5) we have found additional bosons with correct total number of bosons.

In (4.15) the quasi-Nambu-Goldstone bosons in the $U(k)$ gauge theory are decomposed into first two elements $\mathbf{C}^{k}$ and $\mathbf{C}^{\frac{1}{2} k(k-1)}$. The first one $\mathbf{C}^{k}$ corresponds to (approximate) Nambu-Goldstone bosons for translational symmetry broken by a configuration of vortices. One of them $\mathbf{C}$ is an exact Nambu-Goldstone boson corresponding to the center of mass, if we consider the spacetime symmetry also. The moduli metric for this Nambu-Goldstone boson is flat and forms a direct product with other moduli. The translation of each individual vortex around the center of mass becomes a symmetry only asymptotically in the limit of large separations between vortices. It is a parameter of the solution (moduli), since the translational symmetry is valid except finite regions of relative distances and it is spontaneously broken. These relative translation bosons $\mathbf{C}^{k-1}$ are not exact NambuGoldstone bosons, and are called a quasi-Nambu-Goldstone boson. The moduli metric for these bosons $\mathbf{C}^{k-1}$ is not a direct product with others, but sit in a fiber as we will soon see. The second factor $\mathbf{C}^{\frac{1}{2} k(k-1)}$ in Eq. (4.15) corresponds to quasi-Nambu-Goldstone bosons which are accompanied by the Nambu-Goldstone bosons $S U(N)_{\mathrm{G}+\mathrm{F}} /[S U(N-$ $\left.k) \times U(1)^{k}\right]$ for internal symmetry broken by orientation of vortices in the internal space. They are required by the unbroken SUSY and were missed in the purely field theoretical consideration (4.5) in the last subsection.

An advantage of the vortex theory (4.10) is that it can describe the configurations of coincident or nearly coincident vortices without any particular Ansatz. Therefore we can cover values of the orientational moduli (quasi-Nambu-Goldstone bosons) which are not covered by the Ansatz (4.3). Let us consider a generic situation where some of vortices are coincident at several points $z_{I}$. If some of $z_{I}$ coincide, the unbroken symmetry is enhanced and the number of Nambu-Goldstone bosons is reduced. Since the total number of massless bosons is unchanged, the number of quasi-Nambu-Goldstone bosons increases accordingly. Let $n_{a}\left(a=1, \cdots, m ; \sum_{a=1}^{m} n_{a} \leq k\right)$ of them coincide as in (4.7)

$$
Z=\operatorname{diag} .(\underbrace{z_{(1)}, \cdots, z_{(1)}}_{n_{1}} ; \cdots ; \underbrace{z_{(m)}, \cdots, z_{(m)}}_{n_{m}} ; z_{(m+1)} ; z_{(m+2)} ; \cdots ; z_{\left(k-\sum_{a=1}^{m} n_{a}+m\right)}) .
$$

These VEVs break the gauge symmetry $U(k)$ down to $\prod_{a=1}^{m} U\left(n_{a}\right) \times U(1)^{k-\sum n_{a}}$. By using similar argument as above, the moduli manifold looks like

$$
\mathcal{M}_{k, N}=\mathbf{C}^{k} \times \mathbf{C}^{\frac{1}{2} k(k-1)+\sum_{a=1}^{m} \frac{1}{2} n_{a}\left(n_{a}-1\right)} \times \frac{S U(N)_{\mathrm{G}+\mathrm{F}}}{S U(N-k) \times \prod_{a} S U\left(n_{a}\right) \times U(1)^{k+m-\sum_{a} n_{a}}} .
$$


We succeeded to obtain the second factor $\mathbf{C}^{\frac{1}{2} k(k-1)+\sum_{a=1}^{m} \frac{1}{2} n_{a}\left(n_{a}-1\right)}$ of quasi-Nambu-Goldstone bosons which was missed in (4.8) in the field theoretical approach in the last subsection. Compared to the coincident vortices in Eq.(4.15), the unbroken symmetry is enhanced and Nambu-Goldstone bosons decreases whereas the total dimension of moduli space is unchanged. Hence some of the Nambu-Goldstone bosons in the case of non-coincident vortices become the quasi-Nambu-Goldstone bosons in the case of (partially) coincident vortices. (This corresponds to singular points in $\mathcal{M}_{k, N} / G$ with $G=S U(N)$ [13].)

The most symmetric point occurs if all of $Z_{i}$ coincide giving $Z=z_{0} \mathbf{1}_{k}$. At this point the formula (4.17) for the moduli space gives

$$
\mathcal{M}_{k, N}=\mathbf{C}^{k} \times \mathbf{C}^{k(k-1)} \times G_{N, k}
$$

with $G_{N, k}$ the complex Grassmann manifold parametrizing the Nambu-Goldstone bosons for internal symmetry (orientational zero modes)

$$
G_{N, k}=\frac{S U(N)_{\mathrm{G}+\mathrm{F}}}{S U(N-k) \times S U(k) \times U(1)} .
$$

In the decompositions (4.15), (4.17) and (4.18) we only mean the direct product locally at a neighborhood of each point in the moduli space. It may not be correct globally. In the case of coincident vortices, however, the manifold is exactly a direct product of $\mathbf{C}$ and a $\mathbf{C}^{k^{2}-1}$ bundle over $G_{N, k}$ denoted by ${ }^{5}$

$$
\mathcal{M}_{k, N}=\mathbf{C} \times\left(\mathbf{C}^{k^{2}-1} \ltimes G_{N, k}\right) .
$$

This can be shown as follows. We should obtain the base manifold, by eliminating the fiber. To see this, we substitute $Z=z_{0} \mathbf{1}_{k}$ into the original Lagrangian (4.10), and then we obtain

$$
\mathcal{L}_{\text {reduced }}=\int d^{4} \theta\left[k z_{0}^{\dagger} z_{0}+\operatorname{tr}\left(\varphi \varphi^{\dagger} e^{V}\right)-c \operatorname{tr} V\right] .
$$

Then $V$ can be eliminated immediately using its algebraic equation of motion as $V=$ $-\log \operatorname{det} \varphi \varphi^{\dagger}$. Substituting this back into (4.21) we obtain

$$
\mathcal{L}_{\text {reduced }}=\int d^{4} \theta\left[k z_{0}^{\dagger} z_{0}+c \log \operatorname{det}\left(\mathbf{1}_{k}+\hat{\varphi} \hat{\varphi}^{\dagger}\right)\right]
$$

where we have fixed the complex gauge symmetry $U(k)^{\mathbf{C}}$ by choosing $\varphi=\left(\mathbf{1}_{k}, \hat{\varphi}\right)$ with $\hat{\varphi}$ a $k \times(N-k)$ matrix chiral superfield. The second part is the well-known Kähler

\footnotetext{
${ }^{5}$ We denote a fiber bundle $M$ with a fiber $F$ and a base $B$ by $M \simeq F \ltimes B$. Putting $F=0$ we get $M \simeq B$.
} 
potential for the complex Grassmann manifold $G_{N, k}$. This is the standard Kähler quotient construction of the Grassmann manifold [33]. Thus we obtain the metric $\mathbf{C} \times G_{N, k}$ in the limit of $\mathbf{C}^{k^{2}-1} \rightarrow 0$. By restoring the fiber $\mathbf{C}^{k^{2}-1}$, we obtain the bundle structure in Eq.(4.20).

\section{Deformation of the Vortex Moduli Metric}

\subsection{Deformation}

It has been found that the Kähler metric on the moduli space obtained in the effective gauge theory approach of Hanany-Tong is in general different from that obtained in the approach of Manton, although they possess the same topology 21]. This discrepancy may be explained by noting that different definitions of effective field are involved in different approaches. In the case of the Nambu-Goldstone bosons, $S$-matrix elements are unambiguously determined as a consequence of the spontaneously broken symmetry. This result is encoded in low-energy theorems, which determine the effective Lagrangians completely, even though possible field redefinitions may change the appearance of the effective Lagrangians. In the case of the quasi-Nambu-Goldstone bosons, $S$-matrix elements cannot be determined by spontaneously broken symmetry alone. The physical consequences such as the $S$-matrix elements explicitly depend on values of quasi-Nambu-Goldstone moduli parameters [12, 13, 14]. Therefore low-energy theorems cannot determine the structure of the effective Lagrangians even after fixing the definition of fields.

Since different effective Lagrangians for the same system share the same symmetry properties, they should be related by deforming the Lagrangians while preserving the symmetry. Therefore we propose to list up all possible terms consistent with symmetry in order to construct the effective Lagrangian. This method should be applicable to solve ambiguities due to field redefinitions, even in the case of the Nambu-Goldstone bosons, but is particularly useful in the case of quasi-Nambu-Goldstone bosons. Different effective Lagrangians with quasi-Nambu-Goldstone bosons obtained in different approaches should be related once the relation between different parametrizations of quasi-Nambu-Goldstone bosons is identified. In our case, we can deform the metric preserving the $S U(N)$ isometry and SUSY. Such a family of metrics is expected to include both the Hanany-Tong metric and the Manton metric. Moreover we have discussed the case where two gauge couplings of $U(1)$ and $S U(N)$ are related as $g^{2}=N e^{2}$ (3.33). This relation is useful to obtain an exact solution by a simple embedding of $U(1)$ vortices. It is, however, desirable to be 
able to treat the case without this relation, letting $g$ and $e$ independent. In such cases, the vortex theory should be deformed accordingly. This deformation is not expected to alter the number of massless bosons or the topology of the moduli space. Hence the deformation that we discuss in this section should also describe such a case.

For definiteness we discuss the case of two vortices $k=2$. In this case we can have two cases with different patterns of symmetry breaking, depending on the relative position of vortices

$$
\mathcal{M}_{k=2, N}=\left\{\begin{array}{l}
\mathbf{C}^{2} \times \mathbf{C} \times \frac{S U(N)}{S U(N-2) \times U(1)^{2}} \\
\mathbf{C}^{2} \times \mathbf{C}^{2} \times G_{N, 2}
\end{array}\right.
$$

where the first case corresponds to separate vortices in Eq.4.15) and the second case to coincident vortices in Eq.(4.18). Since the common real dimension of factors multiplying the homogeneous space is six due to $\mathbf{C}^{3}$, the moduli space is of cohomogeneity six. The cohomogeneity corresponds to the number of zero modes, which do not originate from the genuine Nambu-Goldstone modes, in generic point of moduli space.

To count the degree of freedom for the deformation, we construct invariants of $U(2)_{\mathrm{g}} \times$ $S U(N)_{\mathrm{f}}$. To this end, let us define two by two matrices invariant under the flavor group $S U(N)_{\mathrm{f}}$

$$
M_{1} \equiv \varphi \varphi^{\dagger} e^{V}, \quad M_{2} \equiv Z e^{-V} Z^{\dagger} e^{V}
$$

both of which transform as $M_{a} \rightarrow e^{\Lambda} M_{a} e^{-\Lambda}$. Noting the Cayley-Hamilton theorem $A^{2}-$ $(\operatorname{tr} A) A+(\operatorname{det} A) \mathbf{1}_{2}=0$ for an arbitrary two by two matrix $A$, the independent invariants are found to be

$$
I_{\alpha} \equiv\left\{\operatorname{tr} M_{1}, \operatorname{tr} M_{2}, \operatorname{tr}\left(M_{1}^{2}\right), \operatorname{tr}\left(M_{2}^{2}\right), \operatorname{Re} \operatorname{tr}\left(M_{1} M_{2}\right), \operatorname{Im} \operatorname{tr}\left(M_{1} M_{2}\right)\right\}
$$

Note that the number of invariants, six, coincides with the cohomogeneity of the moduli space $\mathcal{M}_{k=2, N}$. In the $U(k)$ gauge theoretical point of view, the most general Lagrangian is given by

$$
\mathcal{L}_{\text {deformed }}^{k=2}=\int d^{4} \theta\left[f\left(I_{\alpha}\right)-c \operatorname{tr} V\right]
$$

with $f$ an arbitrary function of six arguments.

Further we have to require that the center of positions should decouple from other moduli variables so that $\mathcal{M}_{k, N}=\mathbf{C} \times \hat{\mathcal{M}}_{k, N}$ holds everywhere in the level of the metric. This requires that $Z$ should appear quadratically. We thus obtain

$$
\mathcal{L}_{\text {deformed }}^{k=2}=\int d^{4} \theta\left[\operatorname{tr} M_{2}+g\left(\operatorname{tr} M_{1}, \operatorname{tr}\left(M_{1}^{2}\right)\right)-c \operatorname{tr} V\right]
$$


with $g$ an arbitrary function of two arguments. One can expect that the interactions of the genuine Nambu-Goldstone boson corresponding to the center of mass translation is determined completely. It is perhaps surprising that the interactions of $k-1$ quasi-NambuGoldstone bosons in $Z$ are also determined, even though they are not constrained by lowenergy theorems. Physical reason behind this fact is that the quasi-Nambu-Goldstone bosons become Nambu-Goldstone bosons asymptotically when all vortices are very far away from each other. The Hanany-Tong metric is obtained if we further choose the simplest case $g\left(\operatorname{tr} M_{1}, \operatorname{tr} M_{1}^{2}\right)_{\mathrm{HT}}=\operatorname{tr} M_{1}$. We have not yet identified explicitly the choice corresponding to the approach of Manton.

For $k=1$ we have no degree of freedom for the deformation in agreement with the purely field theoretical argument. ${ }^{6}$ For general $k$ the Lagrangian can be written as

$$
\mathcal{L}_{\text {deformed }}^{k}=\int d^{4} \theta\left[\operatorname{tr} M_{2}+g\left(\operatorname{tr} M_{1}, \operatorname{tr}\left(M_{1}^{2}\right), \cdots, \operatorname{tr}\left(M_{1}^{k}\right)\right)-c \operatorname{tr} V\right]
$$

where the definitions of $M_{1}$ and $M_{2}$ are identical to (5.2), because the Cayley-Hamilton theorem for an arbitrary $k$ by $k$ matrix is the form of $A^{k}-(\operatorname{tr} A) A^{k-1}+\cdots+(\operatorname{det} A) \mathbf{1}_{k}=0$. The Hanany-Tong metric is still given by $g_{\mathrm{HT}}=\operatorname{tr} M_{1}$.

The deformation does not alter the shape of the compact submanifold $G / H$ in the moduli space parametrized by Nambu-Goldstone bosons, but it deforms the shape along noncompact directions parametrized by quasi-Nambu-Goldstone bosons (or radii of NambuGoldstone coset manifolds).

\subsection{Component Lagrangian}

We calculate the bosonic Lagrangian in the case of $k=2$. Superfields are expanded by the components fields (in the Wess-Zumino gauge) as

$$
\begin{aligned}
& \varphi\left(x^{m}, \theta, \bar{\theta}\right)=\varphi+\theta^{2} F, \\
& Z\left(x^{m}, \theta, \bar{\theta}\right)=Z+\theta^{2} F_{Z}, \\
& V\left(x^{m}, \theta, \bar{\theta}\right)=-\theta \sigma^{m} \bar{\theta} v_{m}+\frac{1}{2} \theta^{2} \bar{\theta}^{2} D, \\
& e^{V}\left(x^{m}, \theta, \bar{\theta}\right)=\mathbf{1}_{k}-\theta \sigma^{m} \bar{\theta} v_{m}+\frac{1}{2} \theta^{2} \bar{\theta}^{2}\left(D-\frac{1}{2} v^{2}\right)
\end{aligned}
$$

\footnotetext{
${ }^{6}$ The Kähler potential corresponding to (5.5) includes an arbitrary function $g: \mathcal{L}=\int d^{4} \theta\left[\operatorname{tr} M_{2}+\right.$ $\left.g\left(M_{1}\right)-c V\right]$. However arbitrariness disappears when $V$ is eliminated and the manifold is $\mathbf{C} \times \mathbf{C} P^{N}$ with the Fubini-Study metric [32].
} 
where we have written only bosonic fields. Each invariant can be calculated in terms of component fields, to yield

$$
\begin{aligned}
& \operatorname{tr}\left(M_{1}\right)= \operatorname{tr} \varphi \varphi^{\dagger}-\theta \sigma^{m} \bar{\theta} \operatorname{tr}\left(i \varphi \stackrel{\leftrightarrow}{\partial}_{m} \varphi^{\dagger}+\varphi \varphi^{\dagger} v_{m}\right) \\
&+ \theta^{2} \bar{\theta}^{2} \operatorname{tr}\left[F F^{\dagger}+\frac{1}{4} \square \varphi \varphi^{\dagger}+\frac{1}{4} \varphi \square \varphi^{\dagger}-\frac{1}{2} \partial_{m} \varphi \partial^{m} \varphi^{\dagger}\right. \\
&\left.\quad-\frac{i}{2}\left(\varphi \stackrel{\leftrightarrow}{\partial}_{m} \varphi^{\dagger}\right) v^{m}+\frac{1}{2} \varphi \varphi^{\dagger}\left(D-\frac{1}{2} v^{2}\right)\right], \\
& \operatorname{tr}\left(M_{1}^{2}\right)=\operatorname{tr}\left[\left(\varphi \varphi^{\dagger}\right)^{2}\right]-2 \theta \sigma^{m} \bar{\theta} \operatorname{tr}\left[\varphi \varphi^{\dagger}\left(i \varphi \stackrel{\leftrightarrow}{\partial}_{m} \varphi^{\dagger}+\varphi \varphi^{\dagger} v_{m}\right)\right] \\
&+\theta^{2} \bar{\theta}^{2}\left[2 \operatorname { t r } \left\{\varphi \varphi ^ { \dagger } \left(F F^{\dagger}+\frac{1}{4} \square \varphi \varphi^{\dagger}+\frac{1}{4} \varphi \square \varphi^{\dagger}-\frac{1}{2} \partial_{m} \varphi \partial^{m} \varphi^{\dagger}-\frac{i}{2}\left(\varphi \stackrel{\leftrightarrow}{\partial}_{m} \varphi^{\dagger}\right) v^{m}\right.\right.\right. \\
&\left.\left.\left.\quad+\frac{1}{2} \varphi \varphi^{\dagger}\left(D-\frac{1}{2} v^{2}\right)\right)\right\}-\frac{1}{2} \operatorname{tr}\left(i \varphi \stackrel{\leftrightarrow}{\partial}_{m} \varphi^{\dagger}+\varphi \varphi^{\dagger} v_{m}\right)^{2}\right], \\
&\left.\operatorname{tr}\left(M_{2}\right)\right|_{\theta^{2} \bar{\theta}^{2}}= \operatorname{tr}\left[-\frac{1}{2} \partial_{m} Z^{\dagger} \partial^{m} Z+\frac{1}{4} \square Z^{\dagger} Z+\frac{1}{4} Z^{\dagger} \square Z-\frac{i}{2}\left(Z \stackrel{\leftrightarrow}{\partial}_{m} Z^{\dagger}+Z^{\dagger} \stackrel{\leftrightarrow}{\partial}_{m} Z\right) v^{m}\right. \\
&+\left.\frac{1}{2} Z^{\dagger} v_{m} Z v^{m}-\frac{1}{4}\left(Z^{\dagger} Z+Z Z^{\dagger}\right) v^{2}-\frac{1}{2}\left(Z^{\dagger} Z-Z Z^{\dagger}\right) D+F_{Z}^{\dagger} F_{Z}\right] .
\end{aligned}
$$

with $A \stackrel{\leftrightarrow}{\partial} B \equiv A \partial B-(\partial A) B$. Defining

$$
X^{a} \equiv \operatorname{tr}\left(M_{1}^{a}\right) \equiv C^{a}-\theta \sigma^{m} \bar{\theta} u_{m}^{a}+\frac{1}{2} \theta^{2} \bar{\theta}^{2} E^{a},
$$

we can calculate an arbitrary function of them, to give [32]

$$
g(X)=g(C)-\theta \sigma^{m} \bar{\theta} g_{,_{a}} u_{m}^{a}+\frac{1}{2} \theta^{2} \bar{\theta}^{2}\left[g_{, a}(C) E^{a}-\frac{1}{2} g_{,_{a b}}(C) u^{a} u^{b}\right]
$$

Here ${ }_{a}$ denotes a differentiation with respect to $C^{a}$. Substituting Eq. (5.10) with Eqs. (5.8) into the Lagrangian (5.5), we obtain the component Lagrangian explicitly.

We have constructed the most general form of the effective Lagrangian for non-Abelian vortices which contains the deformation of the moduli metric compatible with SUSY and the global symmetry on the world-volume. We conjecture that the freedom of this deformation resolves the discrepancy between the Hanany-Tong metric and the Manton metric, although it remains to demonstrate this point explicitly.

\section{Discussion}

We have obtained a nonlinear sigma model on vortex world-volume formulated by auxiliary gauge fields. This fact raises a possibility that our model might provide an interesting 
possibility for dynamically induced gauge bosons [9] on the brane. Models of induced gauge bosons have been discussed in a number of different context previously [34, 35]. This type of composite gauge bosons can offer an alternative 36] to the localization problem for the gauge bosons which has been notoriously difficult. In the case of a single vortex $(k=1)$ we have the $\mathbf{C} P^{N}$ model formulated by an auxiliary $U(1)$ gauge field. It is well known that the large- $N$ analysis of the $\mathbf{C} P^{N}$ model displays a dynamically induced $U(1)$ gauge boson as a composite of Nambu-Goldstone bosons provided that the FI-term $c$ is proportional to $N$ [37, 9]. In our case, we have $c \sim 1 / g^{2}=N / g^{2} N$ with $g$ the gauge coupling of the original gauge theory. If one takes the limit of $N \rightarrow \infty$ with $g^{2} N$ fixed, one has a dynamical $U(1)$ gauge boson on a single vortex. So it is weak coupling limit in the original gauge theory. In the case of multi-vortices we will have $U(k)$ gauge fields for coincident vortices and $U(1)^{k}$ gauge fields for separate vortices. It resembles to the situation of D-branes. Thus we may have localized gauge fields on vortices induced by quantum effects. Let us note that this model gives the following interesting problem quantum mechanically. The $\mathbf{C} P^{N}$ model in four dimensions has the so-called sigma-model anomaly proportional to the first Chern class originated from the $U(1)$ gauge field [38]. Presumably this is related with the gauge anomaly in six-dimensions proportional to ${ }^{7} N_{\mathrm{F}}-2 N_{\mathrm{C}}$. We can add matters cancelling anomaly into the four-dimensional $\mathbf{C} P^{N}$ model by starting from the six-dimensional theory with $N_{\mathrm{F}}=2 N_{\mathrm{C}}$. Investigating such a relation of quantum anomaly in four and six dimensions is a very interesting problem to be explored.

Another interesting possibility is the application to composite models of matter fields such as quarks. In four spacetime dimensions, SUSY nonlinear sigma models were proposed as models of composite quarks in which we identify superpartners of NambuGoldstone bosons as quarks [8]. Phenomenological viability has been studied by working out various coset spaces. Previously not much attention have been payed as to the origin of the underlying SUSY linear theory which realizes the appropriate global symmetry that is broken spontaneously. Non-Abelian vortices may provide the origin of these models. We expect that such a problem can be considered realistically by introducing additional matter fields or taking different gauge group in the original gauge theory.

Instead of the $U(N)$ gauge group which gives a coset space $\mathbf{C} P^{N-1}$ for a single vortex, we can consider other gauge groups. A single non-Abelian vortex in the theory with $S O(N) \times U(1)$ gauge group and appropriate number of flavors is expected to give the effective theory with another coset space, the quadric surface $Q^{N-2} \equiv S O(N) /[S O(N-$

\footnotetext{
${ }^{7}$ We would like to thank David Tong for pointing this out.
} 
2) $\times U(1)]$ which is also a Hermitian symmetric space. The sigma model Lagrangian on $Q^{N-2}$ was formulated in [33, 39] as a $U(1)$ SUSY gauge theory by introducing a superpotential $W=\sigma \varphi^{a} \varphi^{a}(a=1, \cdots, N)$ with an auxiliary chiral superfield $\sigma$ with $U(1)$ charge -2 . The equation of motion for $\sigma$ gives a constraint $\varphi^{a} \varphi^{a}=0$ defining $Q^{N-2}$ embedded into $\mathbf{C} P^{N-1}$. For $k$-vortices, the gauge group in the effective theory is extended to $U(k)$, and the matter chiral superfields $\varphi^{a}$ to a $k$ by $N$ matrix $\varphi^{I a}(I=1, \cdots, k)$, similarly to sect 4.2. The auxiliary chiral superfield $\sigma$ may then be extended to a $U(k)$ symmetric tensor $\sigma^{I J}$, which appears in the superpotential ${ }^{8}$ as $W=\operatorname{tr}\left(\sigma \varphi \varphi^{T}\right)$. Other Hermitian symmetric spaces, formulated in 33. as SUSY gauge theories, may also be realized as the effective theory on a single vortex by taking other gauge groups with appropriate number of flavors in the original gauge theory.

In the case of $U(1)$ (Abelian) vortex, it was found that gauge field is localized by warp factors if we couple the theory with gravity 20. We expect that the same mechanism will work for non-Abelian vortices also. Analysis for non-Abelian vortices in $D=6$ SUGRA remains as a future problem.

Both effective Lagrangians constructed in [21] and by the Manton's method [6] include up to two derivatives with respect to world-volume coordinates. They are valid provided zero-mode fluctuations vary only weakly on the world volume : $\partial_{m}$ (fields) $<<1$. In the case of a single ANO vortex $(N=1, k=1)$, the effective action including higher derivative corrections was constructed by using nonlinear realizations and the Green-Schwarz method [19]. They are supersymmetric generalization of the Nambu-Goto action. The action for non-Abelian vortices of the Nambu-Goto type is an interesting open problem.

\section{Acknowledgements}

We would like to thank David Tong for a useful discussion. M.E. gratefully acknowledges support from the Iwanami Fujukai Foundation and from a 21st Century COE Program at Tokyo Tech "Nanometer-Scale Quantum Physics" by the Ministry of Education, Culture, Sports, Science and Technology. The work of M. N. is supported in part by JSPS. This work is supported in part by Grant-in-Aid for Scientific Research from the Ministry of Education, Culture, Sports, Science and Technology, Japan No.13640269 and 16028203 for the priority area "Origin of Mass" (NS).

\footnotetext{
${ }^{8}$ We would like to thank David Tong for clarifying this point.
} 


\section{References}

[1] P. Horava and E. Witten, Nucl. Phys. B460, 506 (1996) arXiv:hep-th/9510209.

[2] N. Arkani-Hamed, S. Dimopoulos and G. Dvali, Phys. Lett. B429, 263 (1998) arXiv:hep-ph/9803315; I. Antoniadis, N. Arkani-Hamed, S. Dimopoulos and G. Dvali, Phys. Lett. B436, 257 (1998) arXiv:hep-ph/9804398.

[3] L. Randall and R. Sundrum, Phys. Rev. Lett. 83, 3370 (1999) hep-ph/9905221; Phys. Rev. Lett. 83, 4690 (1999) arXiv:hep-th/9906064.

[4] E. Witten and D. Olive, Phys. Lett. B78, 97 (1978).

[5] S. Dimopoulos and H. Georgi, Nucl. Phys. B193, 150 (1981); N. Sakai, Z. f. Phys. C11, 153 (1981); E. Witten, Nucl. Phys. B188, 513 (1981); S. Dimopoulos, S. Raby, and F. Wilczek, Phys. Rev. D24, 1681 (1981).

[6] N. S. Manton, Phys. Lett. B110, 54 (1982); N. S. Manton and J. M. Speight, Commun. Math. Phys. 236, 535 (2003) arXiv:hep-th/0205307.

[7] S. R. Coleman, J. Wess and B. Zumino, Phys. Rev. 177, 2239 (1969); C. G. Callan, S. R. Coleman, J. Wess and B. Zumino, Phys. Rev. 177, 2247 (1969).

[8] W. Buchmuller, S. T. Love, R. D. Peccei and T. Yanagida, Phys. Lett. B115, 233 (1982); W. Buchmuller, R. D. Peccei and T. Yanagida, Phys. Lett. B124, 67 (1983); Nucl. Phys. B227, 503 (1983).

[9] M. Bando, T. Kugo and K. Yamawaki, Phys. Rep. 164, 217 (1988).

[10] M. Bando, T. Kuramoto, T. Maskawa and S. Uehara, Phys. Lett. B138, 94 (1984); Prog. Theor. Phys. 72, 313 (1984); Prog. Theor. Phys. 72, 1207 (1984).

[11] W. Buchmuller and W. Lerche, Annals Phys. 175, 159 (1987).

[12] G. M. Shore, Nucl. Phys. B320, 202 (1989); A. C. W. Kotcheff and G. M. Shore, Int. J. Mod. Phys. A4, 4391 (1989).

[13] M. Nitta, Int. J. Mod. Phys. A14, 2397 (1999) [arXiv: hep-th/9805038.

[14] K. Higashijima, M. Nitta, K. Ohta and N. Ohta, Prog. Theor. Phys. 98, 1165 (1997) arXiv:hep-th/9706219. 
[15] E. Abraham and P. K. Townsend, Phys. Lett. B 291, 85 (1992); J. P. Gauntlett, R. Portugues, D. Tong, and P. K. Townsend, Phys. Rev. D63, 085002 (2001) arXiv:hep-th/0008221; J. P. Gauntlett, D. Tong, and P. K. Townsend, Phys. Rev. D63, 085001 (2001) arXiv:hep-th/0007124; Phys. Rev. D64, 025010 (2001) arXiv:hep-th/0012178; D. Tong, Phys. Rev. D66, 025013 (2002) arXiv:hep-th/0202012; JHEP 0304, 031 (2003) arXiv:hep-th/0303151; K. S. M. Lee, Phys. Rev. D67, 045009 (2003) arXiv:hep-th/0211058; M. Arai, M. Naganuma, M. Nitta and N. Sakai, Nucl. Phys. B652, 35 (2003) arXiv:hep-th/0211103; in Garden of Quanta - In honor of Hiroshi Ezawa, World Scientific Publishing Co. Pte. Ltd., Singapore arXiv:hep-th/0302028; M. Shifman and A. Yung, Phys. Rev. D67, 125007 (2003) arXiv:hep-th/0212293; M. Arai, E. Ivanov and J. Niederle, Nucl. Phys. B680, 23 (2004) arXiv:hep-th/0312037; K. Kakimoto and N. Sakai, Phys. Rev. D68, 065005 (2003) arXiv:hep-th/0306077; Y. Isozumi, K. Ohashi and N. Sakai, JHEP 0311, 061 (2003) arXiv:hep-th/0310130; JHEP 0311, 060 (2003) arXiv:hep-th/0310189; Y. Isozumi, M. Nitta, K. Ohashi and N. Sakai, arXiv:hep-th/0404198, to appear in Phys. Rev. Lett; hep-th/0405194, to appear in Phys. Rev. D; arXiv:hep-th/0405129.

[16] A. A. Abrikosov, Sov. Phys. JETP 5, 1174 (1957) [Zh. Eksp. Teor. Fiz. 32, 1442 (1957)], [Reprinted in Solitons and Particles, Eds. C. Rebbi and G. Soliani (World Scientific, Singapore, 1984), p. 356].

[17] H. B. Nielsen and P. Olesen, Nucl. Phys. B61, 45 (1973), [Reprinted in Solitons and Particles, Eds. C. Rebbi and G. Soliani (World Scientific, Singapore, 1984), p. $365]$.

[18] H.J. de Vega and F.A. Schaposnik, Phys. Rev. D14, 1100 (1976).

[19] J. Hughes and J. Polchinski, Nucl. Phys. B278, 147 (1986); J. Hughes, J. Liu and J. Polchinski, Phys. Lett. B180, 370 (1986).

[20] A. Chodos and E. Poppitz, Phys. Lett. B471, 119 (1999) arXiv:hep-th/9909199; T. Gherghetta and M. E. Shaposhnikov, Phys. Rev. Lett. 85, 240 (2000) arXiv:hep-th/0004014; I. Oda, Phys. Lett. B496, 113 (2000) arXiv:hep-th/0006203; Phys. Rev. D62, 126009 (2000) arXiv:hep-th/0008012; E. Ponton and E. Poppitz, JHEP 0102, 042 (2001) arXiv:hep-th/0012033; M. Giovannini, H. Meyer and M. E. Shaposhnikov, Nucl. Phys. B619, 615 
(2001) arXiv:hep-th/0104118; M. Giovannini, Phys. Rev. D66, 044016 (2002) arXiv:hep-th/0205139; M. Giovannini, J. V. Le Be, S. Riederer, Class. Quant. Grav. 19, 3357 (2002) arXiv:hep-th/0205222; B. de Carlos and J. M. Moreno, JHEP 0311, 040 (2003) arXiv:hep-th/0309259.

[21] A. Hanany and D. Tong, JHEP 0307, 037 (2003) arXiv:hep-th/0306150.

[22] R. Auzzi, S. Bolognesi, J. Evslin, K. Konishi and A. Yung, Nucl. Phys. B673, 187 (2003) arXiv:hep-th/0307287.

[23] D. Tong, Phys. Rev. D69, 065003 (2004) arXiv:hep-th/0307302.

[24] R. Auzzi, S. Bolognesi, J. Evslin and K. Konishi, Nucl. Phys. B 686, 119 (2004) arXiv:hep-th/0312233.

[25] M. Shifman and A. Yung, Phys. Rev. D 70, 025013 (2004) arXiv:hep-th/0312257.

[26] M. Shifman and A. Yung, Phys. Rev. D 70, 045004 (2004) arXiv:hep-th/0403149.

[27] A. Hanany and D. Tong, JHEP 0404, 066 (2004) arXiv:hep-th/0403158.

[28] M. A. C. Kneipp and P. Brockill, Phys. Rev. D 64, 125012 (2001), M. A. C. Kneipp, Phys. Rev. D 68, 045009 (2003), M. A. C. Kneipp, Phys. Rev. D 69, 045007 (2004), M. A. C. Kneipp, arXiv:hep-th/0401234.

[29] E. J. Weinberg, Phys. Rev. D19, 3008 (1979); Phys. Rev. D24, 2669 (1981).

[30] N. Arkani-Hamed, T. Gregoire and J. Wacker, JHEP 0203, 055 (2002) arXiv:hep-th/0101233.

[31] N. Marcus, A. Sagnotti and W. Siegel, Nucl. Phys. B224, 159 (1983).

[32] K. Higashijima and M. Nitta, Prog. Theor. Phys. 103, 833 (2000) arXiv:hep-th/9911225.

[33] K. Higashijima and M. Nitta, Prog. Theor. Phys. 103, 635 (2000) arXiv:hep-th/9911139.

[34] K. Akama, Lect. Notes Phys. 176, 267 (1982), arXiv:hep-th/0001113.

[35] G. R. Dvali, G. Gabadadze and M. A. Shifman, Phys. Lett. B497, 271 (2001) arXiv:hep-th/0010071. 
[36] E. Kh. Akhmedov, Phys. Lett. B521, 79 (2001) arXiv:hep-th/0107223.

[37] A. D'Adda, P. Di Vecchia and M. Lüscher, Nucl. Phys. B152, 125 (1979); E. Witten, Phys. Rev. D16, 2991 (1977).

[38] G. W. Moore and P. Nelson, Phys. Rev. Lett. 53, 1519 (1984).

[39] K. Higashijima, T. Kimura, M. Nitta and M. Tsuzuki, Prog. Theor. Phys. 105, 261 (2001) arXiv:hep-th/0010272. 